\title{
The Role of Nerve- versus Muscle-Derived Factors in Mammalian Neuromuscular Junction Formation
}

\author{
Shuo Lin, ${ }^{1}$ Lukas Landmann, ${ }^{2}$ Markus A. Ruegg, ${ }^{1}$ and Hans Rudolf Brenner ${ }^{3}$ \\ ${ }^{1}$ Biozentrum and Institutes of ${ }^{2}$ Anatomy and ${ }^{3}$ Physiology, Department of Biomedicine, University of Basel, CH-4056 Basel, Switzerland
}

\begin{abstract}
Neuromuscular junctions (NMJs) normally form in the central region of developing muscle. In this process, agrin released from motor neurons has been considered to initiate the formation of synaptic acetylcholine receptor (AChR) clusters (neurocentric model). However, in muscle developing in the absence of nerves and thus of agrin, AChR clusters still form in the muscle center. This raises the possibility that the region of NMJ formation is determined by muscle-derived cues that spatially restrict the nerve to form synapses from aneural AChR clusters, e.g., by patterned expression of the agrin receptor MuSK (muscle-specific kinase) (myocentric model). Here we examine at initial stages of synaptogenesis whether the responsiveness of myotubes to agrin is spatially restricted, whether the regions of NMJ formation in wild-type muscle and of aneural AChR cluster formation in agrin-deficient animals correlate, and whether AChR cluster growth depends on the presence of agrin. We show that primary myotubes form AChR clusters in response to exogenous agrin in their central region only, a pattern that can spatially restrict NMJ formation. However, the nerve also makes synapses in regions in which aneural AChR clusters do not form, and agrin promotes synaptic cluster growth from the first stages of neuromuscular contact formation. These data indicate that aneural AChR clusters per se are not required for NMJ formation. A model is proposed that explains either the neurocentric or the myocentric mode of NMJ formation depending on a balance between the levels of MuSK expression and the availability of nerve-released agrin.
\end{abstract}

Key words: agrin; MuSK; synapse formation; development; neuromuscular junction; muscle

\section{Introduction}

Reciprocal interactions driving the formation of synapses between neurites and their target cells have been investigated intensively at the neuromuscular junction (NMJ). Until recently, agrin, a heparansulfate proteoglycan that is secreted from motor neurons and activates the receptor tyrosine kinase MuSK (muscle-specific kinase) in the muscle fiber (DeChiara et al., 1996; Gautam et al., 1996), has been considered the primary organizer of subsynaptic muscle differentiation (McMahan, 1990; Bezakova and Ruegg, 2003). In this view, synaptic acetylcholine receptor $(\mathrm{AChR})$ clusters only form on muscle fibers in response to nerve-derived agrin (neurocentric model of synapse formation). This model has been challenged by the observation that AChR clusters form independently of motor neurons in the central region of developing mouse diaphragm muscle in which synapses are normally made (Thaler et al., 1999; Lin et al., 2001; Yang et al., 2001). These findings raise two important questions. First, are myotubes only receptive to innervation in their central region (e.g., as a result of a spatially restricted responsiveness to agrin)?

Received Dec. 18, 2007; revised Feb. 11, 2008; accepted Feb. 13, 2008.

This work was supported by grants from the Swiss National Science Foundation, the Swiss Foundation for Research on Muscle Diseases, and the Kanton Basel-Stadt. We thank Dr. Clarke Slater for advice on this manuscript and Drs. T. M. DeChiara, J. R. Sanes, and S. Arber for providing musk ${ }^{+/-}$, agrn ${ }^{+/-}$, and $\mathrm{Hb}^{\text {nlslacZ }}$ mutants, respectively.

Correspondence should be addressed to Hans Rudolf Brenner, Institute of Physiology, Department of Biomedicine, University of Basel, Klingelbergstrasse 50, CH-4056 Basel, Switzerland. E-mail: hans-rudolf.brenner@unibas.ch.

DOI:10.1523/JNEUROSCI.5590-07.2008

Copyright $\odot 2008$ Society for Neuroscience $\quad$ 0270-6474/08/283333-08\$15.00/0
Second, do motor neurons contact aneural AChR clusters to form new synapses (myocentric model) (Kummer et al., 2006), or can motor neurons induce postsynaptic differentiation independently of preexisting aneural clusters as observed in cultured myotubes (Anderson and Cohen, 1977; Frank and Fischbach, 1979) and during ectopic synapse formation in adult muscle (Lomo, 2003)? In support of the myocentric concept pioneer motor neurons in the zebrafish preferentially contact AChR clusters that form aneurally in adaxial slow muscle fibers and incorporate them into postsynaptic specializations (Flanagan-Steet and Sanes, 2005; Panzer et al., 2006). Conversely, in the laterforming fast fibers, AChR clusters form after the arrival of motor neurons, consistent with the neurocentric model.

Because motor innervation in zebrafish is regulated somewhat differently from that in mammals (Lefebvre et al., 2007), it is important to ask which model is implicated in mammalian muscle. Because live imaging of motor neurites in the process of making contacts with muscle fibers is not possible in embryonic mammalian muscle, this issue must be addressed more indirectly in mammals. To this end, we have asked, (1) where do AChR clusters form in developing mouse diaphragm myotubes when recombinant agrin is applied along the entire length of the myotubes, and (2) where do axons make new synapses on myotubes in wild-type diaphragms, in relation to the region in which aneural AChR clusters form in mutants lacking agrin. The results show, first, that the limits of the synaptic region can be explained by the spatially restricted responsiveness of myotubes to agrin and, second, that the formation of synapses is not dependent on 
preexisting aneural AChR clusters. Furthermore, the decrease in AChR cluster formation observed in $m u s k^{+/-}$mutants expressing low levels of MuSK can be compensated by agrin overexpression by muscles or nerves. The present and previous findings combined are consistent with the idea that the mode of neuromuscular synapse formation, i.e., myocentric versus neurocentric, in a given muscle will depend on the levels of agrin and MuSK autoactivation at the center of the myotube.

\section{Materials and Methods}

Animals. Experiments were done on mutant and wild-type (w.t.) mouse embryos at embryonic day 12.5 (E12.5) to E15.5. Mutants used were musk $^{-1-}$ (DeChiara et al., 1996), agrn $^{-1-}$ (Lin et al., 2001), Hb9 ${ }^{-1-}$ derived from $H b 9^{\text {nlslacZ }}$ (Arber et al., 1999), and Hb9-agrn (Ksiazek et al., 2007), expressing full-length chick agrin in motor neurons under the control of the promoter of $\mathrm{Hb} 9$, a transcription factor expressed specifically in motor neurons. $M C K-\mathrm{cmag}_{B 8}$ mutants expressing a chick miniagrin $\left(\mathrm{cN} 257 \mathrm{C} 21 \mathrm{~B}_{8}\right)$ selectively in muscle under the control of the muscle creatine kinase (MCK) promoter will be described in detail elsewhere (S. Lin, H. R. Brenner, and M. A. Ruegg, unpublished observations). Synapse and AChR cluster formation were analyzed in homozygous mutants, with heterozygous and w.t littermates serving as controls, and in hemizygous mutants with w.t. littermates as controls, respectively.

For timed matings, males and females were kept in separate compartments of the same cage for $3 \mathrm{~d}$. The separation was then removed at 3:00 P.M. of the third day, and mating was checked by the presence of a copulation plug at 8:00 A.M. the following morning, when the animals were separated. Pregnancies were then dated as E0.5. Given the mixed genetic background of the mice used and to avoid variations in the results attributable to different mating times within a nominal stage of development, we used littermates only for comparison of AChR cluster numbers and innervation between different genotypes at a given stage.

Culture conditions. For culturing E14.5 diaphragms, the thorax was excised from the embryos, and liver, lungs, and heart were removed to ensure adequate oxygenation by diffusion. The diaphragms were left suspended by the ribcage to prevent damage of the muscle fibers and were maintained in medium M199 supplemented with 5\% horse serum and penicillin/streptomycin in an atmosphere of $95 \% \mathrm{O}_{2}$ and $5 \% \mathrm{CO}_{2}$ at $37^{\circ} \mathrm{C}$, in the presence or absence of chicken mini-agrin $\mathrm{cN} 25_{7} \mathrm{c} 21 \mathrm{~B}_{8}$ (Meier et al., 1998).

Immunohistochemistry. Embryos were decapitated and fixed in $4 \%$ paraformaldehyde in PBS. Diaphragm muscles were dissected and postfixed for $1 \mathrm{~h}$, washed for $20 \mathrm{~min}$ in $0.1 \mathrm{M}$ glycine in PBS, permeabilized, blocked for $1 \mathrm{~h}$ in $0.5 \%$ Triton X-100, 20\% goat serum in PBS, and incubated overnight with rabbit antibodies against neurofilaments (1: 1000; Millipore Bioscience Research Reagents, Temecula, CA) and with mouse monoclonal antibody against synaptophysin (1:200; Dako North America, Carpinteria, CA) and washed three times for $60 \mathrm{~min}$ in $0.5 \%$ Triton X-100 in PBS, all at $4^{\circ} \mathrm{C}$. After three 20 min washes in $0.5 \%$ Triton $\mathrm{X}-100$ in PBS, muscles were incubated with $\alpha$-bungarotoxin-Alexa-594 (1:1000; Invitrogen, Carlsbad, CA) and with goat anti-mouse and antirabbit secondary antibodies each coupled to Alexa-488 (1:1000; Invitrogen) for $45 \mathrm{~min}$ in $0.5 \%$ Triton X-100 in PBS, washed three times for 60 min in $0.5 \%$ Triton X-100 in PBS, all at room temperature, and mounted in Prolong Gold (Invitrogen). Specimens were stored at $-20^{\circ} \mathrm{C}$.

Image acquisition and processing. Diaphragms were analyzed with a confocal laser scanning microscope (TCS 4D; Leica Microsystems, Mannheim, Germany) equipped with an argon/krypton laser, an acousto-optical tunable filter module and operating in the simultaneous acquisition mode. Preliminary experiments demonstrated that this procedure resulted in crosstalk that did not exceed 3\%. In general, images were recorded with PlanFluotar $16 \times /$ numerical aperture (NA) 0.5 or PlanApo $40 \times / \mathrm{NA} 1.25$ objective lenses, which results in a $z$-resolution of 2.5 and $0.4 \mu \mathrm{m}$. Accordingly, image stacks were sampled with 2 and 0.5 $\mu \mathrm{m} z$-steps, respectively. Diaphragms at E12.5 were imaged with PlanApo $63 \times / \mathrm{NA} 1.4$, and single synapses at E15.5 with PlanApo $100 \times /$ NA 1.4 objective lenses, respectively, both with $0.1 \mu \mathrm{m} z$-steps.

During NMJ development, AChRs first appear as microclusters that coalesce into larger clusters at later stages. For analysis of AChR cluster number, size, and nerve-muscle contact formation during progressive stages of development, image stacks were filtered with a $5 \times 5 \times 5$ median filter to reduce background noise, stray light (Landmann and Marbet, 2004), and AChR microclusters. The threshold intensity for recognition of a cluster after filtering by the software in any given image stack was then set in such a way that only clusters observed by visual inspection in at least three consecutive optical sections were detected. Trials to shift the threshold up to $15 \%$ above or below the level obtained as described above did not significantly affect the measured values of AChR cluster number and size. Subsequently, every optical section of a stack was analyzed with the colocalization module of the Imaris software package (Bitplane, $\mathrm{Zu}$ rich, Switzerland). Determination of thresholds for colocalization, a crucial step, has been described previously (Landmann and Marbet, 2004) and, to avoid subjective bias, was monitored by the automatic thresholding device based on an algorithm excluding intensity pairs that exhibit no correlation (Pearson's coefficient $<0$ ) (Costes et al., 2004). The software rated AChR clusters as being contacted by nerves when the fluorescence signals colocalized in the same voxel of $\sim 0.3 \times 0.3 \times 0.5 \mu \mathrm{m}$ in size. This dimension only reflects the actual resolution approximately because images were filtered to avoid false-positive colocalization signals caused by background noise. Because application of a filter kernel "dissipates" not only noise but also signal, the technique trades in some loss of resolution for eradication of false-positive colocalization. Nevertheless, comparison of image stacks before and after filtering showed that the loss in resolution was minor in the present context (supplemental Fig. 1, available at www.jneurosci.org as supplemental material). The distribution of fluorescent signals was visualized by maximum intensity projection (MIP) or surface rendering (IsoSurface, Imaris) using the thresholds that have been applied to colocalization analysis.

Quantitative analysis of AChR cluster bands and nerve branches. For estimating the width of the band of AChR clusters in the center of the diaphragm, MIPs were made from image stacks taken with the $16 \times / 0.5$ NA objective in three adjacent $600 \mu \mathrm{m}$ windows around the point of nerve entry (or around the region of widest cluster band in nerve-free $\mathrm{Hb}^{-1-}$ mutants). For each stack, the area of the diaphragm region comprising AChR clusters and its length were then determined as outlined in supplemental Figure 2 (available at www.jneurosci.org as supplemental material), using the measuring tool of ImageAccess. The average width of the AChR cluster band of a stack was calculated by dividing its area by its length. Finally, the AChR cluster band widths of the three adjacent stacks from each hemidiaphragm were averaged. The average width of the cluster band obtained from each homozygous mutant in this way was expressed relative to band widths obtained from all heterozygous and w.t. embryos of the same litter. ImageAccess was also used for analyzing nerve branching. Quantitative data are means \pm SE. They were tested for significant differences by two-tailed $t$ tests.

\section{Results}

To study the relationship between nerves and AChR clusters, optical sections were made through the entire thickness (10-25 $\mu \mathrm{m}$ depending on age) of diaphragms that had been stained to visualize nerves and AChR clusters. Because myotubes near the point of nerve entry develop and become innervated earlier than those located more distally (Babiuk et al., 2003), an analysis window, defined relative to the point of nerve entry, was used to compare muscles at different stages of development. This window was $720 \mu \mathrm{m}$ when muscles were imaged at $40 \times$ and $1800 \mu \mathrm{m}$ when imaged at $16 \times$ (see Figs. 2, 5).

\section{Spatial development of AChR clusters in wild-type mice}

As a basis of our comparison of agrin-dependent versus agrinindependent AChR cluster formation in developing diaphragms, we started by making a detailed morphological analysis of nerveinduced and aneural AChR cluster formation in wild-type mice.

At E12.5, trunks of the phrenic nerve run over the developing diaphragm, extending ventrally well into the presumptive muscle 

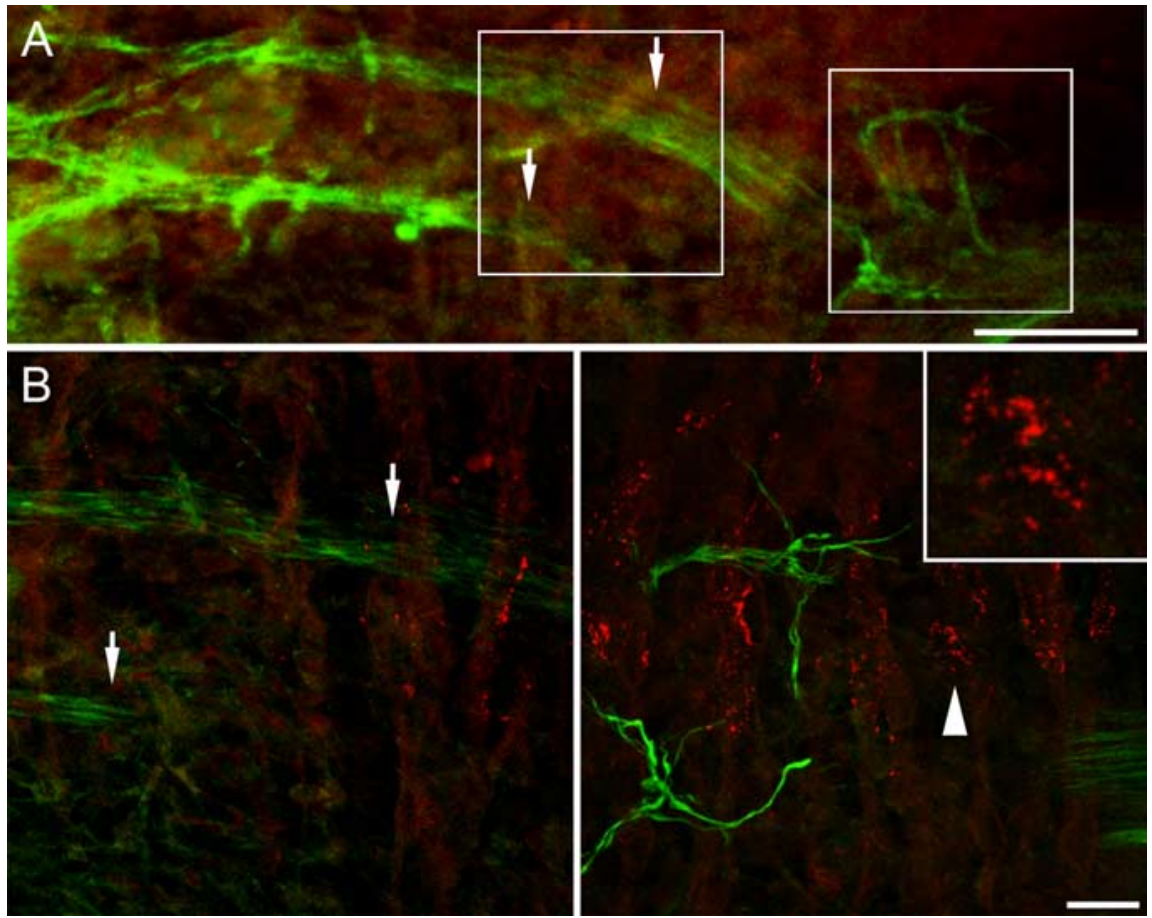

Figure 1. Wild-type mouse diaphragm at E12.5. Maximum intensity projection of stacks of images taken at $2 \mu \mathrm{m}(\boldsymbol{A})$ and 0.1 $\mu \mathrm{m}(\boldsymbol{B})$ intervals. Speckles of AChRs are present on the myotubes near but not distant from the area of nerve entry. They run in a band centered beneath the nerve, but none are in contact with it. Left and right frames in $A$ denote the areas enlarged in left and right bottom $\boldsymbol{B}$, respectively. Arrows mark nerve trunks in $\boldsymbol{A}$ and $\boldsymbol{B}$, left. Close-up inset in $\boldsymbol{B}$, right, shows microclusters grouped but not fused to a single AChR cluster (arrowhead). Scale bars: $\boldsymbol{A}, 70 \mu \mathrm{m} ; \boldsymbol{B}$ and inset, 20 and $7 \mu \mathrm{m}$, respectively. not observed in $\mathrm{agrn}^{-/-}$and $\mathrm{Hb}^{-/-} \mathrm{mu}-$ tants (see below) (Fig. 3B).

At E14.5, AChR clusters are larger and often located along the nerves (Fig. $2 B$ ). Most microclusters have disappeared (supplemental Fig. 1B, available at www. jneurosci.org as supplemental material). The central band of AChR clusters is considerably wider than at E13.5 (Fig. 2, compare $A, B)$, and $\sim 35 \%$ are contacted by motor axons (Fig. 3A). Overall, AChR cluster volumes are larger at this stage than at E13.5, now averaging $\sim 230 \mu \mathrm{m}^{3}$. The average volume of clusters contacted by neurons was $\sim 400 \mu \mathrm{m}^{3}$, i.e., $25 \%$ greater than at E13.5 (Fig. 3B).

Between E14.5 and E15.5, the incidence of nerve contacts and the size of nervecontacted AChR clusters increase only moderately in relation to total AChR cluster number, perhaps because this is the stage when secondary myotubes may begin to form aneural AChR clusters (Harris et al., 1989). The most conspicuous difference is that some postsynaptic sites are now contacted by more than one axon, thus marking the onset of polyneuronal innervation that is well known to occur transiently during early stages of muscle innervation (supplemental Fig. 3, available at www.jneurosci.org as supplemental material). region, with some branching occurring proximally where the nerve enters. Occasional accumulations of AChR microclusters are seen in the region in which the nerve runs but only in proximal and not in distal myotubes. None of these clusters was contacted by nerve processes (Fig. 1).

At E13.5, microclusters have become more numerous, and many appear to have coalesced to form larger clusters, similar to those shown in the inset in Figure 1 and supplemental Figure $1 \mathrm{~A}$ (available at www.jneurosci.org as supplemental material). Nerve branches are more numerous than at E12.5, particularly in the proximal region. The main nerve trunk runs near a central band of small AChR clusters, averaging $50-60 \mu \mathrm{m}^{3}$ in volume (note that surface rendering from image stacks results in volumes rather than en face surfaces for expression of AChR cluster size). Some of the branches reach beyond the central band of AChR clusters into the lateral region of myotubes that is devoid of AChR clusters (Fig. 2A). Because the region responding to agrin by AChR cluster formation is wider than the central band of AChR clusters at this stage (supplemental Fig. 2, available at www. jneurosci.org as supplemental material), the absence of clusters in the lateral regions implies that neurons extending into this region do not secrete agrin. Only $\sim 5 \%$ of all AChR clusters are contacted by axons at this stage (Fig. $3 A$ ). The volume of clusters in direct contact with the nerve is $\sim 320 \mu \mathrm{m}^{3}$, i.e., approximately six times larger than that of the noncontacted clusters (Fig. $3 B$ ). In contrast, the volume of aneural AChR clusters is similar to that in nerve-free $\mathrm{Hb}^{-1-}$ mutants of the same age (Fig. $3 \mathrm{~B}$ ). This indicates, therefore, that NMJ formation is initiated in a small fraction of myotubes at approximately E13.5 and that neuronal contact promotes cluster growth from the earliest stages of synapse formation. This growth appears to require agrin because it is

\section{Responsiveness of myotubes to agrin is limited to their central regions}

To examine the mechanism that confines NMJ formation to the central muscle region, we isolated diaphragms at E14.5 and cultured them with or without $10 \mathrm{~nm}$ recombinant chicken miniagrin $\mathrm{cN} 25_{7} \mathrm{C} 21 \mathrm{~B}_{8}$, a truncated form of chick neural agrin sufficient to cluster AChRs both in vitro (Gesemann et al., 1995) and in vivo (Meier et al., 1998). In the absence of agrin, many or all AChR clusters disappeared within $18 \mathrm{~h}$ of culturing (compare Figs. $2 B, 4 A$ ), and only remnants of degenerating axons could be resolved. The remaining AChR clusters were often present in a narrow band, consistent with the notion that they had already been induced or stabilized by agrin secreted from the earliest axons to branch off the nerve trunk [see neuromuscular contacts (marked yellow) at E13.5 in Fig. 2 A; supplemental Fig. $2 \mathrm{D}$ (available at www.jneurosci.org as supplemental material) ]. The disappearance of AChR clusters was not caused by damage to the fibers because they contracted spontaneously at the end of the culture period (data not shown). We conclude, therefore, that, unlike the situation in the adult, in which synaptic AChR accumulations are stable for months after denervation (Frank et al., 1975; Herczeg et al., 1995), the continued presence of nerves is required in embryonic muscle for AChR cluster maintenance. In contrast, when agrin was added to the medium, AChR clusters were present along a narrow band in the central region of the muscle (Fig. $\left.4 A^{\prime}\right)$. Importantly, no AChR clusters formed in the lateral myotube regions, although the myotubes were accessible to agrin along their entire length. These data indicate that myotubes at E14.5 are only responsive to agrin in their central regions.

A similar result was obtained from E13.5 and E14.5 diaphragms exposed in vivo to mini-agrin $\mathrm{cN} 25_{7} \mathrm{C} 21 \mathrm{~B}_{8}$ expressed by 
myotubes from a transgene under the control of an MCK promoter fragment (MCK$\mathrm{cmag}_{B 8}$ mutant). Although mini-agrin was expressed along the entire length of the myotubes (Fig. 4B), AChR clusters developed only in their central regions (E13.5) (Fig. $4 B^{\prime}$ ) (supplemental Fig. 2, available at www.jneurosci.org as supplemental material), as observed in the cultured diaphragms exposed to agrin. Importantly, the band of AChR clusters induced by mini-agrin was wider than that induced by the nerve in wild-type animals (mean $\pm \mathrm{SE}$, $232 \pm 22 \mu \mathrm{m}, n=3$ vs $164 \pm 4 \mu \mathrm{m}, n=3$; $p<0.01)$. When combined, these findings show that myotubes at this stage are responsive to agrin near their center only, suggesting that the spatial restriction of innervation to the central muscle region results from a spatially restricted responsiveness of the myotubes to agrin. They further show that the region adjacent to the central cluster band, which is normally devoid of aneural AChR clusters, is responsive to agrin as early as E13.5.

To resolve the role of nerve-secreted agrin in synaptic development, we next compared the spatial pattern of AChR cluster formation in wild-type muscle with that of aneural AChR clusters developing in the absence of agrin, i.e., in agrn $^{-1-}$ and $\mathrm{Hb}^{-1-}$ mutants.

\section{Spatial development of AChR clusters in agrn $^{-/-}$and $\mathrm{Hb9}^{-/-}$mutants}

If nerve contacts are made on preformed AChR clusters, as predicted by the myocentric model, then NMJs should be formed exclusively within the central region of the myotubes in which AChR clusters form independently of nerves. To test this prediction, we compared the width of the AChR cluster band at E14.5 in homozygous agrn $^{-/-}$mice with that in control littermates. In the control diaphragms, the band is almost twice as wide as in the littermates lacking agrin (Fig. 5).

Similar reasoning also predicts a difference in the width of the AChR cluster band between mutants lacking phrenic nerves, attributable to deletion of the transcription factor Hb9 (Arber et al., 1999; Thaler et al., 1999), and their control littermates. However, because the nerve secretes factor(s) dispersing AChR clusters (Yang et al., 2001), the difference between nerve-free and innervated diaphragms may be smaller than in the case of the $a g r n^{-/-}$ mutants. Consistent with this idea, cluster bands were narrower in aneural $\mathrm{Hb}^{-/-}$diaphragms than in innervated littermates, although the difference was less pronounced than in the $\mathrm{agrn}^{-/-}$ mutants. When combined, these findings indicate that, in the myotube region adjacent to the aneural AChR clusters, synaptic AChR clusters must have formed de novo in response to agrin secreted by the nerve.

Whether synapses in the central muscle region originate from aneural clusters is not clear. If motor axons contact them preferentially, the number of nerve-associated clusters should also in-
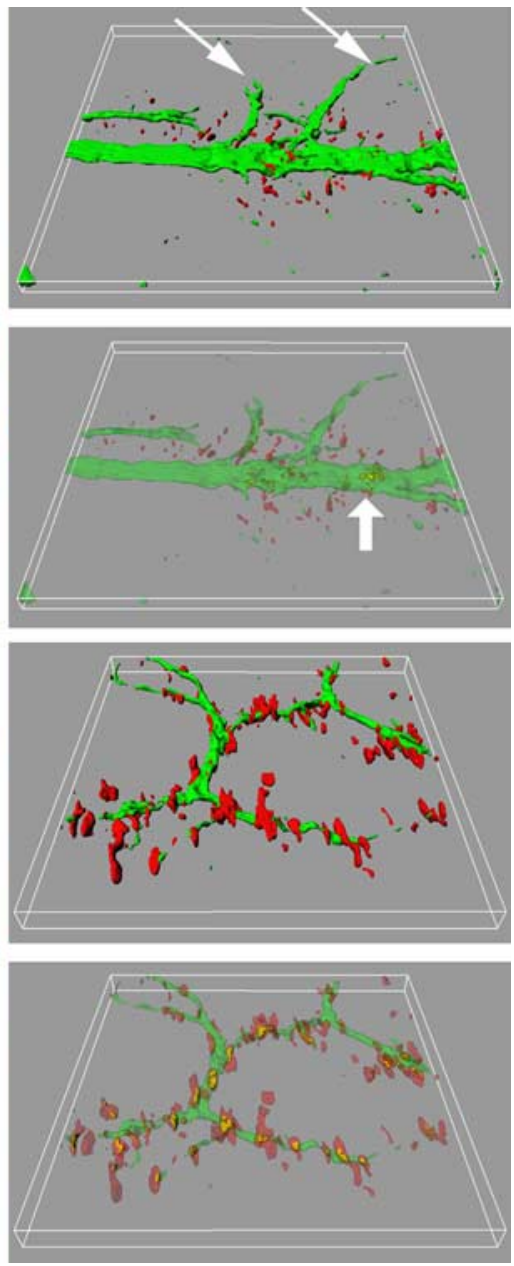

Figure 2. Wild-type mouse diaphragms at E13.5 $(\boldsymbol{A})$ and E14.5 $(\boldsymbol{B})$. In three consecutive frames of $240 \times 240 \mu \mathrm{m}$ in the vicinity of the nerve entry, stacks of images were taken at $0.5 \mu \mathrm{m}$ intervals, and maximum intensity projections (left) and

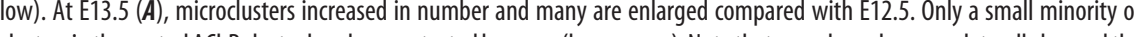
in the central AChR cluster band are contacted by nerve (large arrow). Note that some branches grow laterally beyond the AChR cluster band (small arrows). At E14.5 (B), the band of AChR patches is significantly wider and AChR patches are significantly larger than at E13.5. Numerous clusters are contacted by axons (yellow). Scale bar, $30 \mu \mathrm{m}$.

crease, at least transiently, in $\mathrm{agrn}^{-/-}$mice before the clusters are dispersed. However, the fraction of nerve-associated AChR clusters in $\mathrm{agrn}^{-1-}$ mutants at E14.5 was similar (1.6 $\pm 1.0 \%, 12$ stacks from 3 animals) to that in wild-type mice at E13.5 (2.8 \pm $0.7 \%$ ) (Fig. $3 A$ ), whereas in control littermates, it was similar to E14.5 wild-type mice. Therefore, preferential contact of aneural clusters is unlikely but not excluded by these experiments.

The validity of comparing the widths of AChR cluster bands between mutants and control littermates depends on the assumption that microclusters of AChRs that might have been present in the region more lateral to the larger $\mathrm{AChR}$ clusters were not eliminated by our filtering procedure. For example, if microclusters developed more laterally than the larger AChR clusters in agrn ${ }^{-/-}$mutants, the AChR cluster band widths would be underestimated by filtering. To address this problem, we compared maximum intensity projections from image stacks taken at $40 \times$ of $\mathrm{agrn}^{-/-}$diaphragms at E14.5 before and after median filtering. This comparison showed that there were no microclusters present at this stage outside of the region containing large AChR clusters. Therefore, our comparisons of cluster band widths were not 

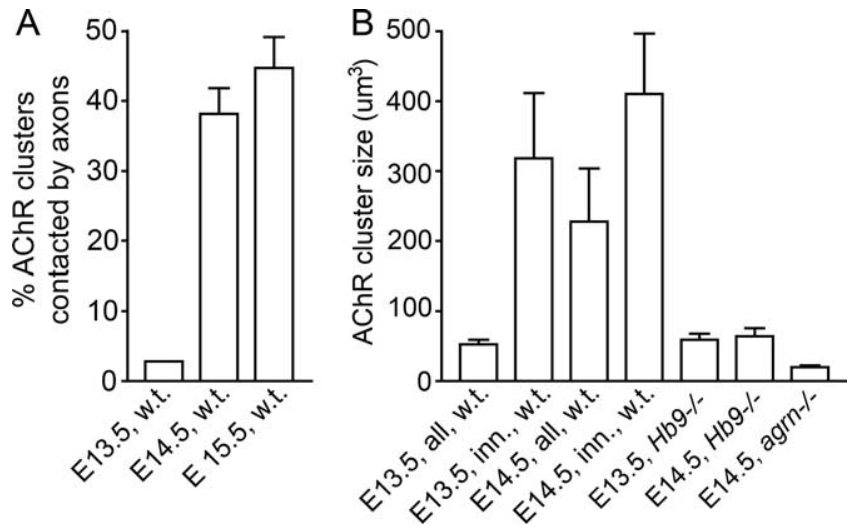

Figure 3. Numbers of AChR clusters contacted by nerves (in percentage of all clusters; $\boldsymbol{A}$ ) and sizes of AChR clusters between E13.5 and E15.5 (B). Data from image stacks as illustrated in Figure 2. Note that the number of AChR clusters contacted by axons and their size increase strongly during this period. In contrast, no contacts are made when nerves do not express agrin, nor do clusters grow in the absence of nerves $\left(\mathrm{Hb}^{-/-}\right)$or agrin $\left(\mathrm{agrn}^{-/}\right)$. Data on agrin mutants are from littermates. Each column gives mean \pm SE from 11-16 stacks analyzed, taken from three to four animals, except $\mathrm{Hb}^{-1-}$ (3-6 stacks, 2 animals), each stack containing 51-556 AChR clusters.

affected by filtering (supplemental Fig. 1, available at www. jneurosci.org as supplemental material).

\section{Overexpression of agrin increases AChR cluster number in musk $^{+/-}$mutants}

The molecular mediators that limit AChR clusters to the central region of myotubes in aneural muscles are not known. However, it is clear that both the responsiveness of the myotubes to agrin (Fig. 4) and the expression of MuSK mRNA in aneural muscles (Kim and Burden, 2008) are high in the central myotube region. Furthermore, the number of aneural AChR clusters is a steep function of the MuSK expression level (Lin et al., 2001). Thus, an attractive explanation is that autoactivation of MuSK, and the nerve-independent clustering of AChRs that results from it, is greatest in the most central region of the myotube. In contrast, in more lateral myotube regions, in which the level of MuSK would be lower, agrin from nerves would be required for AChR cluster formation.

This notion is supported by a number of additional experimental findings. First, in musk $^{+/-}$muscles, the decrease in AChR clustering attributable to the lowering of MuSK level is compensated by agrin overexpressed in motor nerves (Fig. 6). Specifically, we compared AChR cluster numbers in E14.5 muscles from musk $^{+/+}$(wild type) and musk $^{+/-}$mice and in musk $^{+/-}$mice that expressed a chick agrin in their motor neurons (Hb9-agrn; $\left.m u s k^{+/-}\right)$. Whereas AChR cluster number within the test area (compare Figs. 2, 3) was significantly lower in $m u s k^{+/-}$than in musk $k^{+/+}$diaphragms, this difference was not observed in $m u s k^{+/-}$animals that expressed chick agrin. Second, in myotubes expressing mini-agrin $\left(M C K-c m a g_{B 8}\right)$, the central cluster band was significantly wider than in the w.t. mice, at both E13.5 (see above) (supplemental Fig. 2, available at www.jneurosci.org as supplemental material) and E14.5 (data not shown). Therefore, the MCK-driven mini-agrin transgene induced AChR clusters adjacent to the central endplate band. As noted above, however, no clusters formed in the lateralmost myotube regions (Fig. $\left.4 A^{\prime}-C^{\prime}\right)$. When combined, the results of these experiments indicate that agrin overexpression, by either nerves or muscle, can compensate for the reduction of AChR clusters caused by low MuSK expression levels.
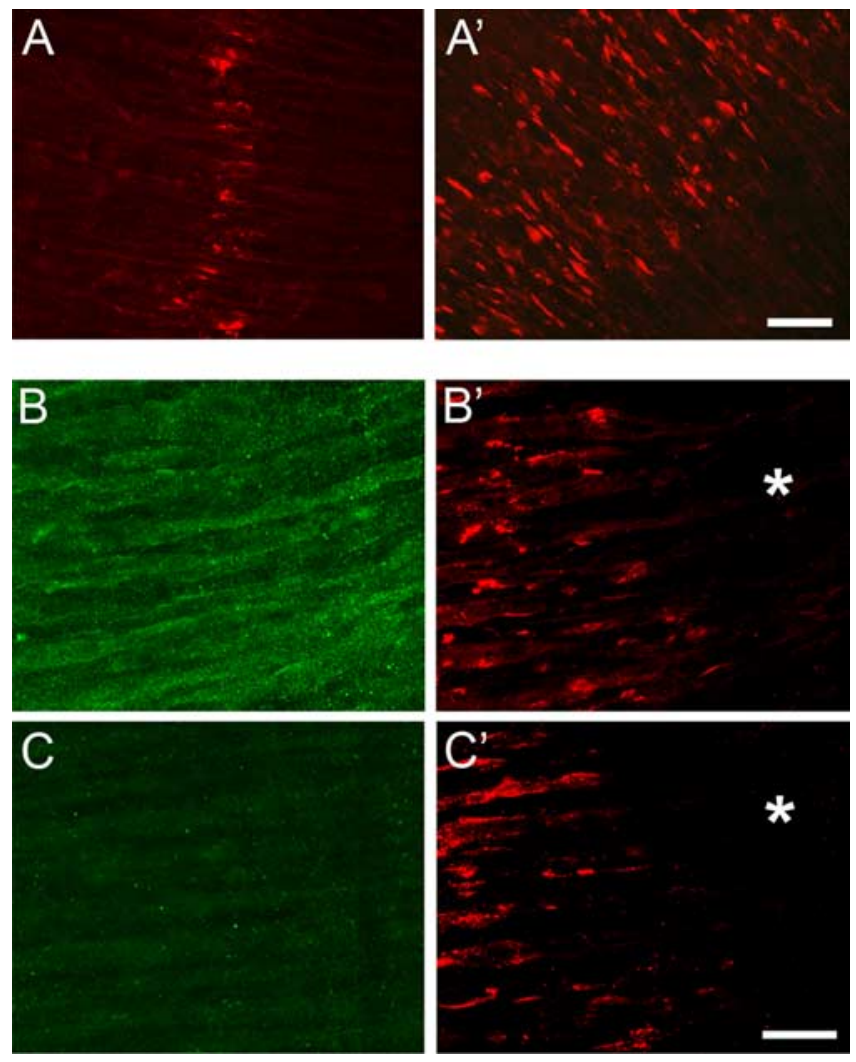

Figure 4. The responsiveness of myotubes in E14.5 diaphragms to agrin is restricted to their central regions. $\boldsymbol{A}, \boldsymbol{A}^{\prime}$, E14.5 diaphragms cultured for $18 \mathrm{~h}$ in the absence $(\boldsymbol{A})$ and presence $\left(\boldsymbol{A}^{\prime}\right)$

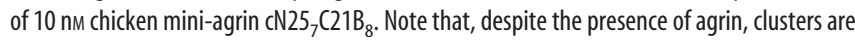
confined to the central region of the myotubes. Scale bar, $40 \mu \mathrm{m} . \boldsymbol{B}, \boldsymbol{B}^{\prime}$, Diaphragms of $M C K-$ $\mathrm{Cmag}_{B 8}$ mice expressing mini-agrin from myotubes in vivo at E13.5. $\boldsymbol{B}$, After staining for agrin immunoreactivity; $\boldsymbol{B}^{\prime}$, same region stained for $A C h R$ clusters. Note absence of $A C h R$ clusters in lateral regions $\left(^{*}\right)$ of the diaphragm despite uniform agrin transgene expression along entire myotubes. $\boldsymbol{C}, \boldsymbol{C}^{\prime}$, Same as in $\boldsymbol{B}$ but from w.t. littermate. Scale bar, $25 \mu \mathrm{m}$.

Finally, while confirming that MuSK is required for aneural AChR clusters to form (data not shown), we observed that the outgrowth and branching of axons from the main nerve trunks was approximately three times stronger in musk $^{-/-}$mutant than in wild-type diaphragms at E13.5, i.e., before many synapses form. This indicates that surface MuSK inhibits the outgrowth of motor neurites in vivo in a way that is independent of its mediation of the formation of AChR clusters (supplemental Fig. 4, available at www.jneurosci.org as supplemental material), as observed previously with motor neurons in culture (Dimitropoulou and Bixby, 2005).

\section{Discussion}

The present results are summarized in Figure $7 A$, which illustrates the relation of nerves to AChR clusters between E12.5 and E14.5 in wild-type and in agrn $^{-/-}$mutant mice. We consider four major conclusions that can be drawn from these data.

First, synapse formation in the diaphragm begins in a small fraction of myotubes as early as E13.5. This is indicated by the rare observation of AChR clusters colocalized with nerves and their larger size compared with aneural clusters at this stage. Substantial numbers of myotubes are not innervated until E14.5. This is in good agreement with the onset of NMJ formation resolved electrophysiologically at corresponding developmental stages in rat intercostal muscles (E14.5-E15.5) (Dennis et al., 1981), thus validating our microscopic approach. 

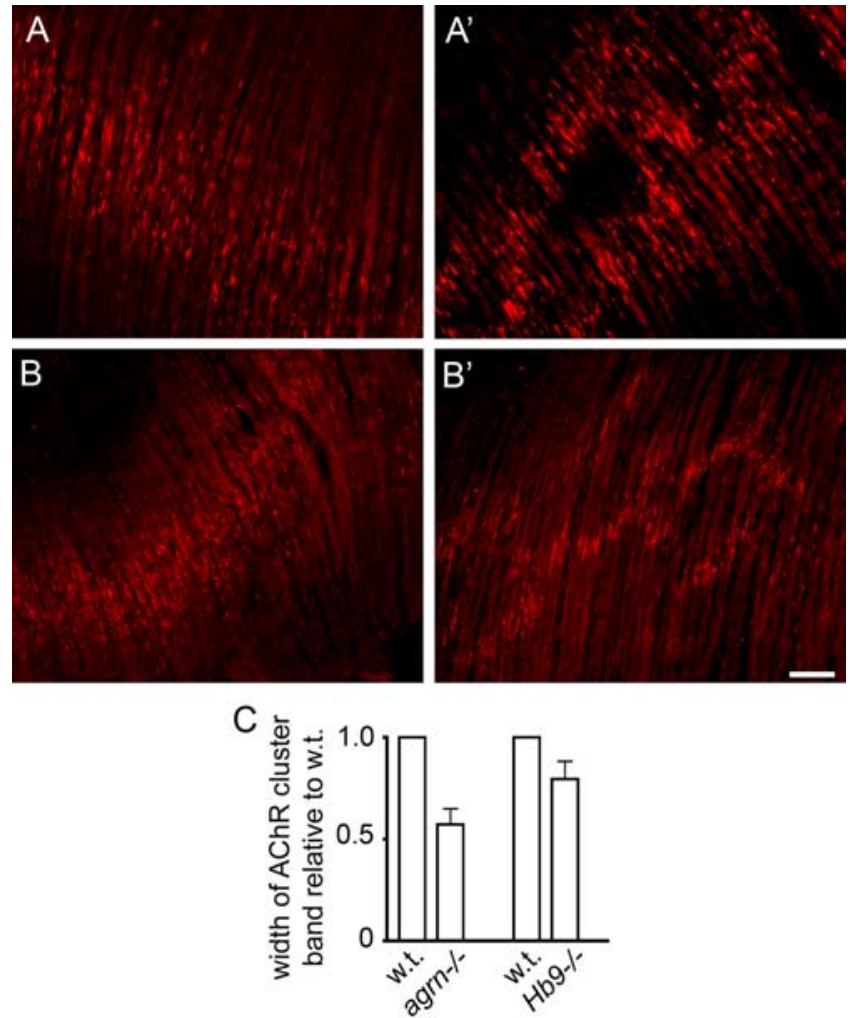

Figure 5. The width of the central band of AChR clusters in diaphragms at E14.5 is narrower in $\operatorname{agrn}^{-1-}(\boldsymbol{A})$ than in control $\left(\boldsymbol{A}^{\prime}\right)$ littermates. A difference is also found between $\mathrm{Hb}^{-1-}$ mutants $(\boldsymbol{B})$ and control $\left(\boldsymbol{B}^{\prime}\right)$ littermates. $\boldsymbol{C}$, Widths of AChR cluster bands (means $\pm \mathrm{SE}$ ) in agrn $^{-/-}$and $\mathrm{Hb}^{-/-}$mutants relative to control littermates. Data are based on five agrn ${ }^{-/-}$ mutants ( 2 litters; $p<0.05$ ) and four $H b 9^{-1-}$ mutants ( 3 litters; $p<0.1$ ). For method, see supplemental Figure 2 (available at www.jneurosci.org as supplemental material). Scale bar, 70 $\mu \mathrm{m}$.

Second, these findings indicate that aneural AChR clusters are not essential for synapse formation. Rather, nerves secreting agrin are required to induce the AChR clusters at the periphery of the NMJ band in w.t. diaphragms, consistent with the agrin hypothesis (McMahan, 1990). At E12.5, although myotubes distant from the point of nerve entry have been reached by the nerve, they have not yet begun to form aneural AChR clusters. At E13.5, branches have reached myotube segments that are outside of the aneural AChR cluster band but are sensitive to agrin. At E14.5, the synaptic band and the agrin-responsive regions are both wider than the aneural AChR cluster band in agrn ${ }^{-/-}$mutants. They are also wider than they were at E13.5. Therefore, by E14.5, synapses have been formed in agrin-responsive segments in which aneural clusters do not form in $\mathrm{agrn}^{-1-}$ mutants.

This conclusion might appear weakened in the face of the relatively small difference in AChR cluster band widths between the $\mathrm{Hb}^{-1-}$ mutants and their controls. It should be noted, however, that $\mathrm{agrn}^{-/-}$mutants are a more valid model than $\mathrm{Hb}^{-/-}$ for investigating whether agrin or aneural AChR clusters are required to initiate synapse formation. Abolishing nerves, as in $\mathrm{Hb}^{-/-}$mice, will not only eliminate agrin secretion but will also reduce nerve-induced electrical activity in the developing myotubes (Dennis et al., 1981), because this can be elicited by evoked and spontaneous release of single ACh quanta (Young and Poo, 1983; Takahashi et al., 2002). Given that, from the earliest stages, normal muscle development is profoundly dependent on functional innervation (Ashby et al., 1993a,b), the developmental state of the muscle and the pattern of aneural AChR clusters will
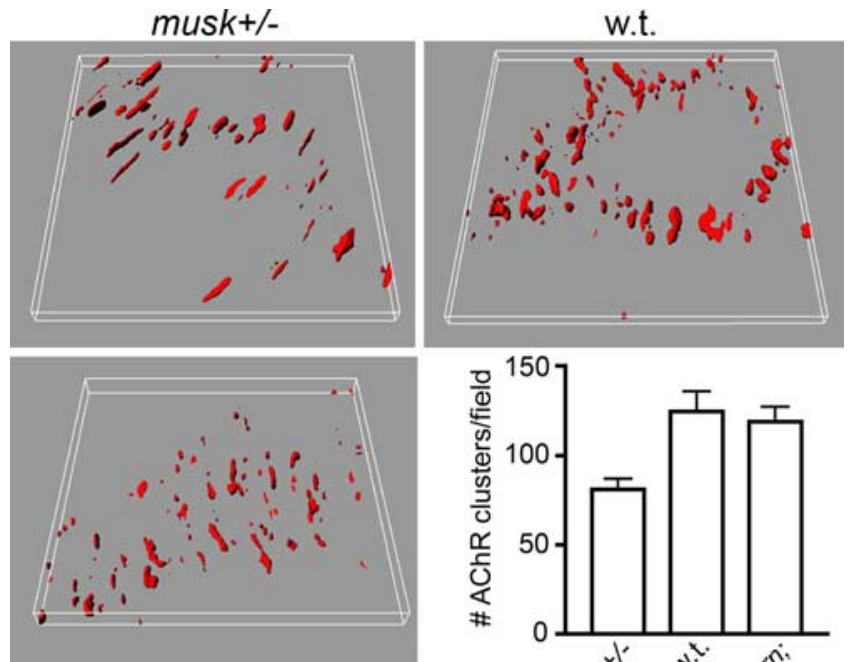

Hb9-agrn;musk+/-



Figure 6. Reduction in AChR cluster number in musk ${ }^{+/-}$mutants is compensated by overexpression of an agrin transgene in motor neurons. All panels are based on data from a single litter. $n=2$ animals each for all genotypes, $11-12$ stacks of optical sections taken at $40 \times$ in 0.5 $\mu \mathrm{m}$ steps from both hemidiaphragms of each animal. Differences in cluster numbers between musk $^{+/-}$versus $\mathrm{Hb} 9$-agrn;musk ${ }^{+/-}$and w.t., respectively, are significant $(p<0.01)$.

be closer to normal in $a g r n^{-1-}$ than in $\mathrm{Hb}^{-/-}$mutants. Moreover, diffusible neural factors such as ACh are known to disperse AChR clusters (Lin et al., 2005; Misgeld et al., 2005). As a consequence, aneural AChR clusters are present in segments of $\mathrm{Hb}^{-/-}$ mutant muscle in which they are not in normal muscle.

In contrast to the present conclusions and in support of a myogenic mode of synapse formation, a central region of elevated AChR $\alpha$ mRNA, coextensive with the aneural AChR cluster band in E18.5 $\mathrm{Hb}^{-1-}$ mutant diaphragms, has been interpreted to reflect synapse-specific transcription of AChR subunit genes (Lin et al., 2001; Yang et al., 2001; Kummer et al., 2006). It should be noted, however, that the central elevation of AChR subunit mRNA in $\mathrm{Hb}^{-/-}$mice at E18.5 does not necessarily reflect synapse-specific transcription of AChR genes. Whereas AChRe mRNA is expressed by a single nucleus at the synapse from an early developmental stage (Brenner et al., 1990; Kues et al., 1995), $\mathrm{AChR} \alpha \mathrm{mRNA}$ is also elevated in distant $(150 \mu \mathrm{m})$ perisynaptic nuclei, some of which are even located outside of the myotubes (Brenner et al., 1990). Given that cultured muscle precursor cells can express AChR mRNA before fusion and that secondary myotubes form at high rate at E18 in the central muscle region (Harris, 1981; Duxson et al., 1989; Harris et al., 1989), the central elevation of $\mathrm{AChR} \alpha$ mRNA in $\mathrm{Hb}^{-/-}$mutants could well be attributable to expression by multiple nuclei of myoblasts and of developing secondary myotubes.

Third, the responsiveness of the diaphragms at E13.5-E14.5 to exogenous agrin is limited to their central regions. Most if not all myotubes at these stages are primary (Harris et al., 1989) and extend well beyond the agrin-induced AChR cluster band. This indicates that primary myotubes are sensitive to agrin in their center but not their periphery. This, together with the fact that presynaptic differentiation is induced by muscle signals secreted in response to MuSK activation (Kim et al., 2008), means that axons will not form synapses outside the central region. This restriction to the center may be further supported by the MuSKdependent inhibition of motor neurite outgrowth mediated by 

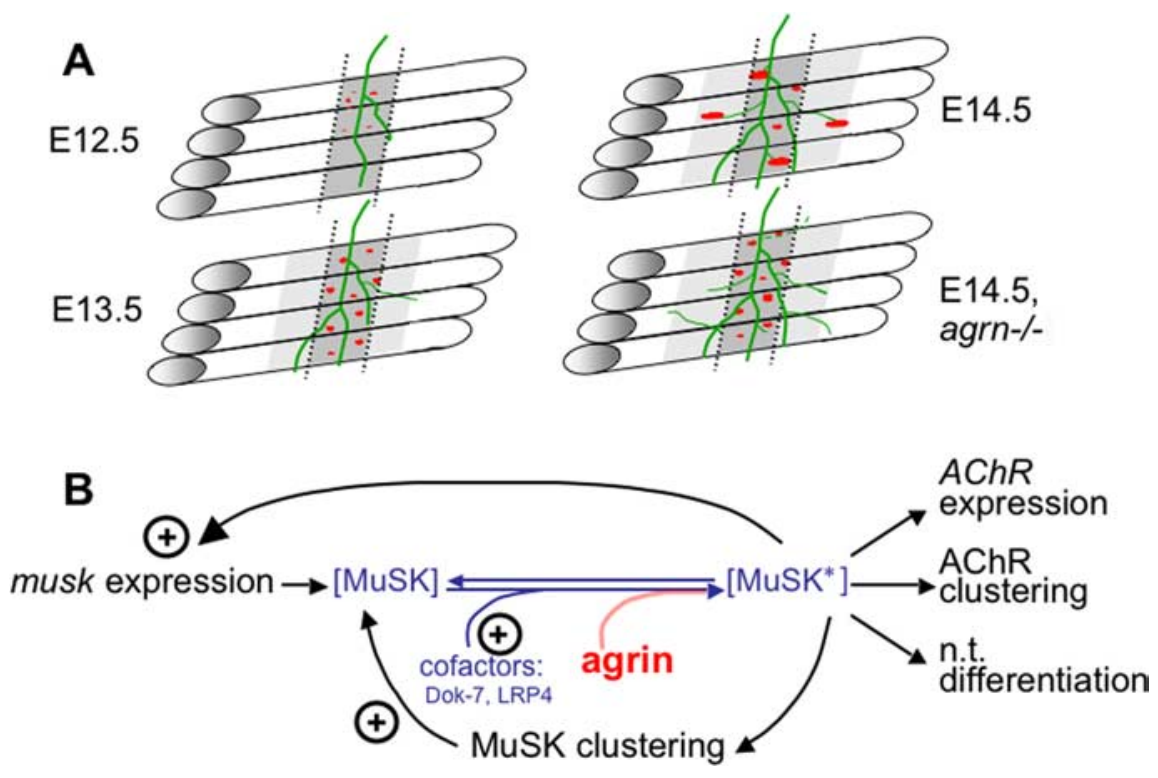

Figure 7. A, Steps of NMJ formation in diaphragms between E12.5 and E14.5 and effects of agrn ablation (bottom right). Dark gray area marked by dotted lines denotes the region in which aneural $A C h R$ clusters form. Adjacent light gray region is responsive to agrin but does not form aneural AChR clusters, perhaps attributable to lower expression levels of MuSK and/or its accessory proteins, e.g., Dok-7 or LRP4. B, Model for early stages of neuromuscular synapse formation. The formation of AChR clusters depends on the local concentration of activated MuSK (MuSK*). In muscle regions in which MuSK is low, agrin from nerves is needed for MuSK activation, resulting in synapse formation consistent with the neurocentric model. Conversely, in regions expressing high levels of MuSK (and of cofactors such as Dok-7 and LRP4), MuSK is autoactivated (MuSK*) and forms numerous aneural AChR clusters. This results in a high probability of motor neurons encountering a preformed AChR cluster, thus favoring innervation of preformed aneural AChR clusters according to the myocentric model. Also shown are feedback loops by which MuSK* stimulates its own aggregation and expression (Jones et al., 1999; Moore et al., 2001).

centrally elevated MuSK (supplemental Fig. 4, available at www.jneurosci.org as supplemental material), although the neurites can, in principle, grow beyond the central region as seen in, for example, musk mutants (DeChiara et al., 1996). Indeed, the high central responsiveness to agrin correlates with the higher expression levels of mRNAs encoding MuSK and its downstream signaling components Dok-7 (downstream of tyrosine kinase 7) and LRP4 (low-density lipoprotein receptor-related protein 4) in the center of the myotube (Okada et al., 2006; Weatherbee et al., 2006; Kim and Burden, 2008). Similarly, because overexpression of Dok-7 increases phosphorylation of MuSK and AChR clustering in cultured myotubes (Okada et al., 2006), centrally increased expression of Dok-7 may account for the presence of aneural $\mathrm{AChR}$ clusters in the central region.

Finally, we propose that the mode of synapse formation implicated in a muscle (myocentric vs neurocentric) depends on the local availability of the MuSK signaling cascade. This view is based on the present findings that (1) exogenous agrin and nerves induce AChR clusters in w.t. myotube segments in which there are no aneural clusters in agrn $^{-/-}$mutants, (2) the decrease in cluster number in $m u s k^{+/-}$mutants is compensated for by agrin transgene expression, and (3) the previous observation (Lin et al., 2001) that AChR clusters are more numerous in innervated (i.e., exposed to agrin from nerves) than in nerve-free diaphragms of musk ${ }^{+/-}$mutants. In the central myotube regions, in which the concentrations of MuSK and its signaling components are sufficient for MuSK autoactivation and aneural AChR cluster formation, the probability that a motor axon will encounter a preformed AChR cluster and become stabilized by activated MuSK at this site will be higher than in regions in which MuSK is low and requires nerve-secreted agrin for activation. Therefore, synapse formation may be myocentric in the central and neurocentric in the adjacent myotube regions, with the myocentric and the neurocentric modes reflecting different states of an equilibrium between inactive MuSK and (agrin- or auto-) activated MuSK${ }^{*}$. This model is consistent with both synapse formation on preformed AChR clusters of zebrafish (Flanagan-Steet et al., 2005) and the formation of ectopic NMJs by foreign nerves transplanted to extrasynaptic regions of adult muscle in which no or only occasional aneural AChR clusters are observed (for review, see Lomo, 2003). A scheme of this model is presented in Figure $7 B$.

\section{References}

Anderson MJ, Cohen MW (1977) Nerve-induced and spontaneous redistribution of acetylcholine receptors on cultured muscle cells. J Physiol (Lond) 268:757-773.

Arber S, Han B, Mendelsohn M, Smith M, Jessell TM, Sockanathan S (1999) Requirement for the homeobox gene $\mathrm{Hb} 9$ in the consolidation of motor neuron identity. Neuron 23:659-674.

Ashby PR, Wilson SJ, Harris AJ (1993a) Formation of primary and secondary myotubes in aneural muscles in the mouse mutant peroneal muscular atrophy. Dev Biol 156:519-528.

Ashby PR, Pinçon-Raymond $\mathrm{M}$, Harris AJ (1993b) Regulation of myogenesis in paralyzed muscles in the mouse mutants peroneal muscular atrophy and muscular dysgenesis. Dev Biol 156:529-536.

Babiuk RP, Zhang W, Clugston R, Allan DW, Greer JJ (2003) Embryological origins and development of the rat diaphragm. J Comp Neurol 455:477-487.

Bezakova G, Ruegg MA (2003) New insights into the roles of agrin. Nat Rev Mol Cell Biol 4:295-308.

Brenner HR, Witzemann V, Sakmann B (1990) Imprinting of acetylcholine receptor messenger RNA accumulation in mammalian neuromuscular synapses. Nature 344:544-547.

Costes SV, Daelemans D, Cho EH, Dobbin Z, Pavlakis G, Lockett S (2004) Automatic and quantitative measurement of protein-protein colocalization in live cells. Biophys J 86:3993-4003.

DeChiara TM, Bowen DC, Valenzuela DM, Simmons MV, Poueymirou WT, Thomas S, Kinetz E, Compton DL, Rojas E, Park JS, Smith C, DiStefano PS, Glass DJ, Burden SJ, Yancopoulos GD (1996) The receptor tyrosine kinase MuSK is required for neuromuscular junction formation in vivo. Cell 85:501-512.

Dennis MJ, Ziskind-Conhaim L, Harris AJ (1981) Development of neuromuscular junctions in rat embryos. Dev Biol 81:266-279.

Dimitropoulou A, Bixby JL (2005) Motor neurite outgrowth is selectively inhibited by cell surface MuSK and agrin. Mol Cell Neurosci 28:292-302.

Duxson MJ, Usson Y, Harris AJ (1989) The origin of secondary myotubes in mammalian skeletal muscles: ultrastructural studies. Development 107:743-750.

Flanagan-Steet H, Fox MA, Meyer D, Sanes JR (2005) Neuromuscular synapses can form in vivo by incorporation of initially aneural postsynaptic specializations. Development 132:4471-4481.

Frank E, Fischbach GD (1979) Early events in neuromuscular junction formation in vitro: induction of acetylcholine receptor clusters in the postsynaptic membrane and morphology of newly formed synapses. J Cell Biol 83:143-158.

Frank E, Gautvik K, Sommerschild H (1975) Cholinergic receptors at denervated mammalian motor end-plates. Acta Physiol Scand 95:66-76.

Gautam M, Noakes PG, Moscoso L, Rupp F, Scheller RH, Merlie JP, Sanes JR (1996) Defective neuromuscular synaptogenesis in agrin-deficient mutant mice. Cell 85:525-535.

Gesemann M, Denzer AJ, Ruegg MA (1995) Acetylcholine receptor- 
aggregating activity of agrin isoforms and mapping of the active site. J Cell Biol 128:625-636.

Harris AJ (1981) Embryonic growth and innervation of rat skeletal muscles. I. Neural regulation of muscle fibre numbers. Philos Trans R Soc Lond B Biol Sci 293:257-277.

Harris AJ, Duxson MJ, Fitzsimons RB, Rieger F (1989) Myonuclear birthdates distinguish the origins of primary and secondary myotubes in embryonic mammalian skeletal muscles. Development 107:771-784.

Herczeg A, Jones G, Brenner HR (1995) Involvement of extracellular matrix in acetylcholine receptor epsilon-subunit gene expression at the rat neuromuscular junction. Neurosci Lett 193:33-36.

Jones G, Moore C, Hashemolhosseini S, Brenner HR (1999) Constitutively active MuSK is clustered in the absence of agrin and induces ectopic postsynaptic-like membranes in skeletal muscle fibers. J Neurosci 19:3376-3383.

Kim N, Burden SJ (2008) MuSK controls where motor axons grow and form synapses. Nat Neurosci 11:19-27.

Ksiazek I, Burkhardt C, Lin S, Seddik R, Maj M, Bezakova G, Jucker M, Arber S, Caroni P, Sanes JR, Bettler B, Ruegg MA (2007) Synapse loss in cortex of agrin-deficient mice after genetic rescue of perinatal death. J Neurosci 27:7183-7195.

Kues WA, Sakmann B, Witzemann V (1995) Differential expression patterns of five acetylcholine receptor subunit genes in rat muscle during development. Eur J Neurosci 7:1376-1385.

Kummer TT, Misgeld T, Sanes JR (2006) Assembly of the postsynaptic membrane at the neuromuscular junction: paradigm lost. Curr Opin Neurobiol 16:74-82.

Landmann L, Marbet P (2004) Colocalization analysis yields superior results after image restoration. Microsc Res Tech 64:103-112.

Lefebvre JL, Jing L, Becaficco S, Franzini-Armstrong C, Granato M (2007) Differential requirement for MuSK and dystroglycan in generating patterns of neuromuscular innervation. Proc Natl Acad Sci USA 104:2483-2488.

Lin W, Burgess RW, Dominguez B, Pfaff SL, Sanes JR, Lee KF (2001) Distinct roles of nerve and muscle in postsynaptic differentiation of the neuromuscular synapse. Nature 410:1057-1064.

Lin W, Dominguez B, Yang J, Aryal P, Brandon EP, Gage FH, Lee KF (2005) Neurotransmitter acetylcholine negatively regulates neuromuscular synapse formation by a Cdk5-dependent mechanism. Neuron 46:569-579.
Lomo T (2003) What controls the position, number, size, and distribution of neuromuscular junctions on rat muscle fibers? J Neurocytol 32:835-848.

McMahan UJ (1990) The agrin hypothesis. Cold Spring Harb Symp Quant Biol 55:407-418.

Meier T, Marangi PA, Moll J, Hauser DM, Brenner HR, Ruegg MA (1998) A minigene of neural agrin encoding the laminin-binding and acetylcholine receptor-aggregating domains is sufficient to induce postsynaptic differentiation in muscle fibres. Eur J Neurosci 10:3141-3152.

Misgeld T, Kummer TT, Lichtman JW, Sanes JR (2005) Agrin promotes synaptic differentiation by counteracting an inhibitory effect of neurotransmitter. Proc Natl Acad Sci USA 102:11088-11093.

Moore C, Leu M, Muller U, Brenner HR (2001) Induction of multiple signaling loops by MuSK during neuromuscular synapse formation. Proc Natl Acad Sci USA 98:14655-14660.

Okada K, Inoue A, Okada M, Murata Y, Kakuta S, Jigami T,Kubo S, Shiraishi H, Eguchi K, Motomura M, Akiyama T, Iwakura Y, Higuchi O, Yamanashi Y (2006) The muscle protein Dok-7 is essential for neuromuscular synaptogenesis. Science 312:1802-1805.

Panzer JA, Song Y, Balice-Gordon RJ (2006) In vivo imaging of preferential motor axon outgrowth to and synaptogenesis at prepatterned acetylcholine receptor clusters in embryonic zebrafish skeletal muscle. J Neurosci 26:934-947.

Takahashi M, Kubo T, Mizoguchi A, Carlson CG, Endo K, Ohnishi K (2002) Spontaneous muscle action potentials fail to develop without fetal-type acetylcholine receptors. EMBO Rep 3:674-681.

Thaler J, Harrison K, Sharma K, Lettieri K, Kehrl J, Pfaff SL (1999) Active suppression of interneuron programs within developing motor neurons revealed by analysis of homeodomain factor HB9. Neuron 23:675-687.

Weatherbee SD, Anderson KV, Niswander LA (2006) LDL-receptor-related protein 4 is crucial for formation of the neuromuscular junction. Development 133:4993-5000.

Yang X, Arber S, William C, Li L, Tanabe Y, Jessell TM, Birchmeier C, Burden SJ (2001) Patterning of muscle acetylcholine receptor gene expression in the absence of motor innervation. Neuron 30:99-410.

Young SH, Poo MM (1983) Spontaneous release of transmitter from growth cones of embryonic neurones. Nature 305:634-637. 